\title{
Robotic natural orifice transluminal endoscopic surgery (R-NOTES) in gynecologic surgeries, a case report and review of literature
}

\begin{abstract}
Background: Natural Orifice Transluminal Endoscopic Surgery (NOTES) involves accessing the abdominal cavity through a natural orifice. It provides easier access for the surgeon; has a reduced recovery times and decreases postoperative pain

Objective: To present an initial experience with robotic natural orifice transluminal surgery (R-NOTES) in gynecology using the da Vinci surgical system.

Case: We present a case of a 40 year old, Asian female, Gravida 2- Para 1-0-1-1 with irregular, heavy menstrual cycle, abnormal uterine bleeding due to uterine leiomyoma (AUB-L) who under went Da Vinci Natural Orifice Transluminal Endoscopic Surgery (NOTES), total Laparoscopic hysterectomy (TLH), bilateral salpingectomy (BS), Lysis of adhesions (LOA), and cystoscopy. The patient had uncomplicated recovery and postoperative care and was discharged on postoperative day zero.
\end{abstract}

Conclusion: Robotic NOTES Total laparoscopic hysterectomy with bilateral salpingectomy is feasible and safe in gynecology patient. This approach has the potential for a less morbid and scarless outcome.

Keywords: da vinci; minimally invasive surgery, natural orifice transluminal endoscopic surgery, robotic NOTES, robotic natural orifice transluminal surgery, single port laparoscopy

\author{
Volume 10 Issue 4 - 2019
}

\section{Shadi Rezai MD,' Richard A Giovane MD, ${ }^{2}$ Savannah N Johnson BS, ${ }^{3}$ Cassandra $\mathrm{E}$ Henderson MD, ${ }^{4}$ Juan Liu MD PhD, ${ }^{5}$ Xiaoming Guan MD PhD' \\ 'Division of Minimally Invasive Gynecologic Surgery, Department of Obstetrics and Gynecology, Baylor College of Medicine, USA ${ }^{2}$ Department of Family Medicine, University of Alabama, USA ${ }^{3}$ School of Medicine, University of Alabama at Birmingham (UAB), USA \\ ${ }^{4}$ Maternal and Fetal Medicine, Department of Obstetrics and Gynecology, Garden OB/GYN At Lake Success, One Hollow Lane, Suite 315, New Hyde Park, New York, I 1042 \\ ${ }^{5}$ The Third Affiliated Hospital of Guangzhou Medical University, China}

Correspondence: Xiaoming Guan MD PhD, Section Chief and Fellowship Director, Division of Minimally Invasive Gynecologic Surgery (MIGS), Department of Obstetrics and Gynecology, Baylor College of Medicine, 665I Main Street, 10th Floor, Houston, Texas, 77030, USA, Tel (832) 826-7464, Fax (832) 825-9349,

Email xiaoming@bcm.edu

Received: June 26, 2019 | Published: August 07, 2019
Abbreviations: MIS, minimally invasive surgery; NOTES, natural orifice transluminal endoscopic surgery; R-NOTES, robotic natural orifice transluminal surgery; SPL, single port laparoscopy; AUB-L, abnormal uterine bleeding due to uterine leiomyoma; TLH, total Laparoscopic hysterectomy; BS, bilateral salpingectomy; LOA, lysis of adhesions

\section{Background}

Natural Orifice Transluminal Endoscopic Surgery (NOTES) involves accessing the abdominal cavity via a natural orifice. ${ }^{1}$ Through NOTES, less tissue is manipulated such as in the case of laparotomy or laparoscopy and with this, it would decrease recovery times as well as have less scaring. ${ }^{1}$ With NOTES being a new technique, success on it relies on the surgeon's comfort level as well as evolving technology to makes this technique easier. ${ }^{2}$

\section{Presentation of the case}

We present a case of a 40-year-old female, Gravida 2, Para 1-01-1, who referred to our clinic for definitive surgical management of dysmenorrhea, irregular heavy menses, pelvic pain, abnormal uterine bleeding secondary to uterine leiomyoma (AUB-L), refractory to medical and conservative surgical management. Her other surgical history is pertinent for a cesarean section and laparoscopic myomectomy. Pelvic exam revealed a $12 \mathrm{~cm}$ retroflexed mobile fibroid uterus.
Pelvic ultrasound showed enlarged leiomyomatous uterus with multiple uterine fibroids, the largest being $4 \mathrm{~cm}$.

Patient underwent Da Vinci Natural Orifice Transluminal Endoscopic Surgery (NOTES), total Laparoscopic hysterectomy (TLH), bilateral salpingectomy (BS), Lysis of adhesions (LOA), and cystoscopy (Figures 1-3).

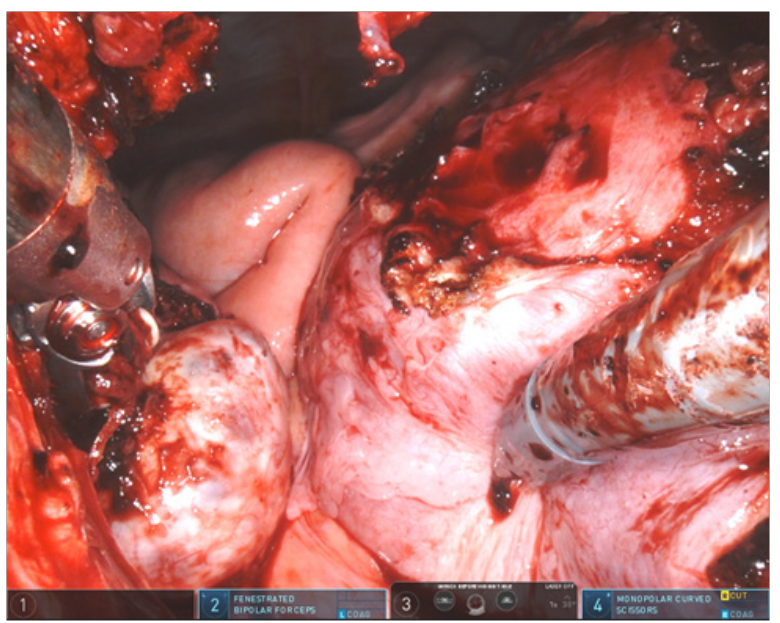

Figure I Performing hysterectomy,Arm 2: fenestrated bipolar grasper,Arm 3: camera, Arm 4: monopolar curved scissors. 


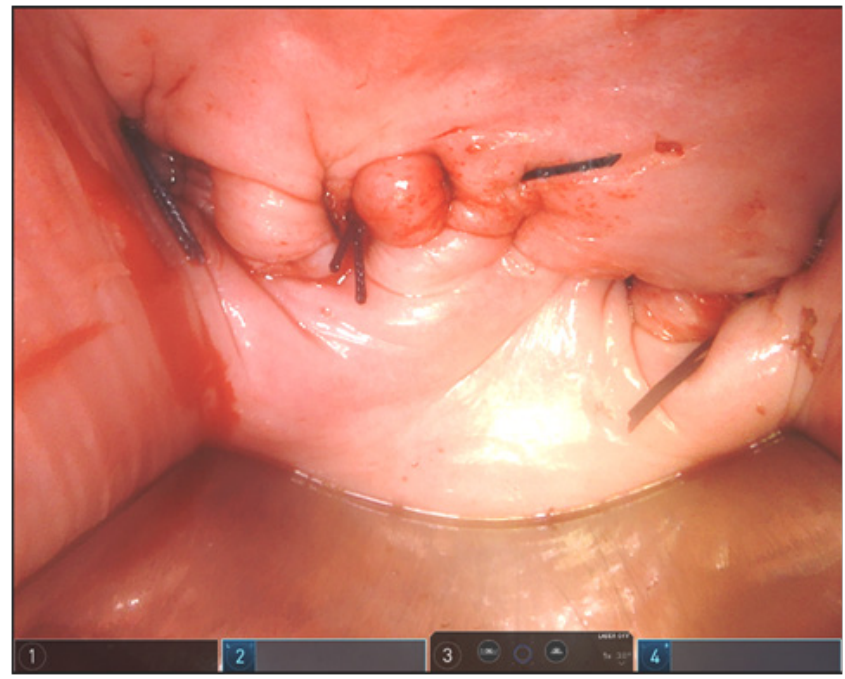

Figure 2 Closed vaginal cuff with V-loc sutures in situ.

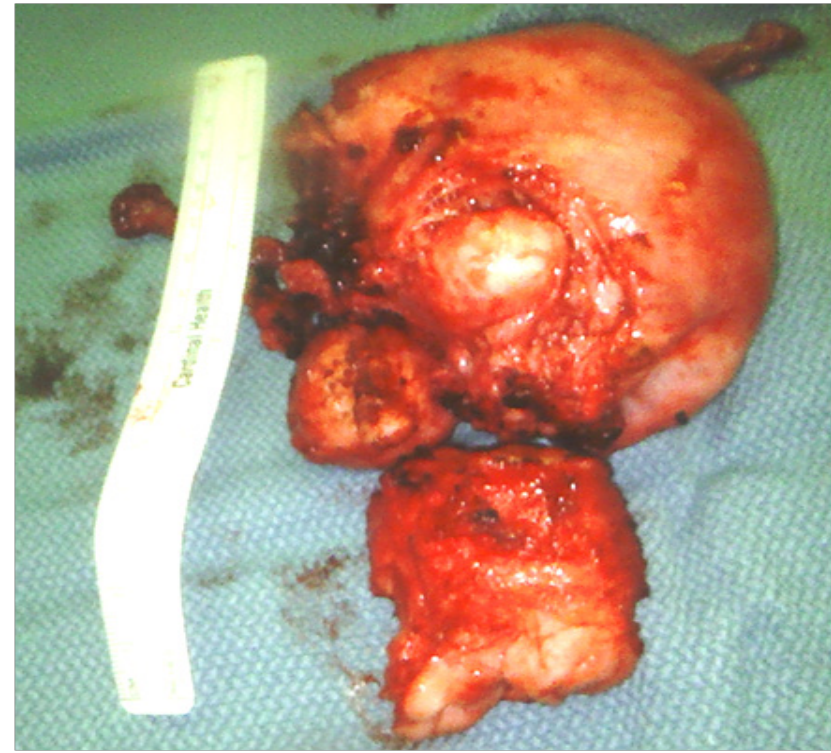

Figure 3 Gross hysterectomy specimen.

GelPOINT mini was placed in the vagina and the Da Vinci XI was docked as following arrangement (Figure 1) (Figure 4):
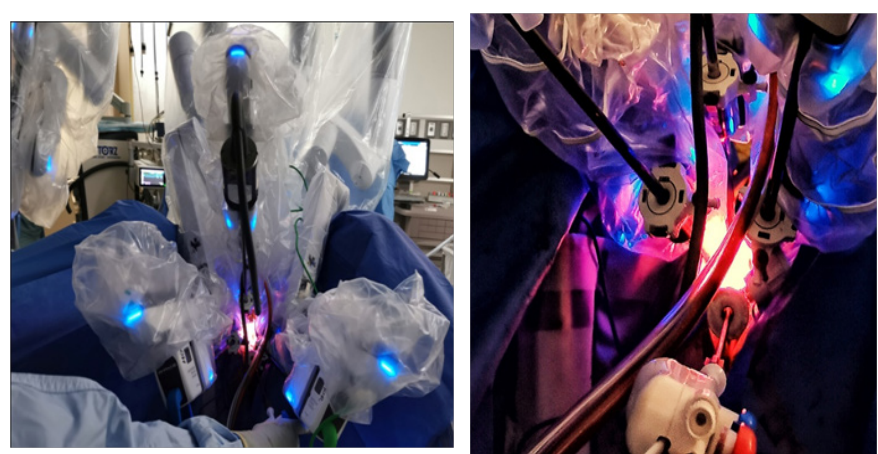

Figure 4 robotic arms setup: Arm 2: fenestrated bipolar grasper, Arm 3: camera, Arm 4: Monopolar curved scissors, $5 \mathrm{~mm}$ air seal used as accessory port, GelPOINT mini in place.
1. Regular robotic instrument and NOT single-site instruments were used.

2. Arm 1: Not Used

3. Arm 2: fenestrated bipolar grasper

4. Arm 3: camera: $10 \mathrm{~mm}, 30$ degree, Bariatric length camera

5. Arm 4: monopolar curved scissor

6. $5 \mathrm{~mm}$ AirSeal placed and used as accessory port, suction was placed through this port

7. The robot was docked in "Thoracic Setting"

8. The patient had uncomplicated recovery and postoperative care and was discharged on postoperative day zero.

\section{Discussions}

Natural Orifice Transluminal Endoscopic Surgery (NOTES) is a technique that uses a natural orifice such as the mouth, anus or vagina in order to access the abdominal cavity. It was first performed by Rao and Reddy in 2006 for an appendectomy ${ }^{3}$ and since then it has been implemented in other surgeries such as gastric and vaginal. Since natural orifices are used, less scarring has been documented as well as less post-operative pain. ${ }^{1,4}$ The use of robotic assistance, such as the Da Vinci machine can aid in dexterity and precision of the surgical process. $^{5}$

Although NOTES has strengths it does have draw backs. Thus far, the safest NOTES route is transvaginally due to enteral closure. ${ }^{1}$ A draw back to this is that it would only benefit female patients. Furthermore, as this technique is new, it is reliant on advances in medical equipment to make this technique easier and more feasible. As there is only one access port, instruments must be positioned properly and multi-tasking can be difficult. ${ }^{6}$

\section{Conclusion}

Robotic NOTES Total laparoscopic hysterectomy with bilateral salpingectomy is feasible and safe in gynecology patient. This approach has the potential for a less morbid and scarless outcome. Further development of robots adaptive to NOTES would boost efforts toward clinical NOTES applications.

\section{Acknowledgments}

Dr. Xiaoming Guan is a speaker for Applied Medical, Rancho Santa Margarita, California.

\section{Conflicts of interest}

Other authors did not report any potential conflicts of interests.

\section{References}

1. Wang X, Meng MQ. Robotics for natural orifice transluminal endoscopic surgery: a review. Journal of Robotics. 2012.

2. Haber GP, Crouzet S, Kamoi K, et al. Robotic NOTES (Natural Orifice Transluminal Endoscopic Surgery) in reconstructive urology: initial laboratory experience. Urology. 2008;71(6):996-1000.

3. Swanstrom LL. Natural orifice transluminal endoscopic surgery. Endoscopy. 2009;41(1):82-85. 
4. Zhou Y, Ren $\mathrm{H}$, Meng MQH, et al. Robotics in natural orifice transluminal endoscopic surgery. Journal of Mechanics in Medicine and Biology. 2013;13(2):1350044.

5. Zhao J, Feng B, Zheng MH, et al. Surgical robots for SPL and NOTES: a review. Minim Invasive Ther Allied Technol. 2015;24(1):8-17.
6. Jayaratna IS, Simma-Chiang V, Desai MM, et al. Robotic NOTES (Natural Orifice Transluminal Endoscopic Surgery) in adults: limitations in the pediatric population. Pediatric Robotic and Reconstructive Urology: A Comprehensive Guide. 2012:229-236. 